Original article

\title{
Association study of polymorphisms within inflammatory genes and methylation status in treatment response in major depression
}

\author{
Metodi Draganov ${ }^{\mathrm{a}}$, María Jesús Arranz ${ }^{\mathrm{b}, * *}$, Juliana Salazarc ${ }^{\mathrm{c}}$, Javier de Diego-Adeliño ${ }^{\mathrm{a}}$, \\ Cristina Gallego-Fabregab ${ }^{b}$, Míriam Jubero ${ }^{a}$, Mar Carceller-Sindreu ${ }^{a}$, Maria J. Portella ${ }^{a, *}$ \\ a Department of Psychiatry, Hospital de la Santa Creu i Sant Pau, Institut d'Investigació Biomèdica Sant Pau (IIB-Sant Pau), Universitat Autònoma de \\ Barcelona (UAB), Centro de Investigación Biomédica en Red de Salud Mental (CIBERSAM), Spain \\ ${ }^{\mathrm{b}}$ Research Laboratory, Fundació Docència i Investigació Mútua Terrassa, Catalonia, Spain \\ ${ }^{\mathrm{c}}$ Department of Genetics, Hospital de la Santa Creu i Sant Pau, IIB-Sant Pau, UAB, CIBER, Spain
}

\section{A R T I C LE I N F O}

Article history:

Received 25 February 2019

Received in revised form 3 May 2019

Accepted 5 May 2019

Available online 14 May 2019

\section{Keywords:}

Treatment response

Major depression

Inflammatory genes

Methylation status

\begin{abstract}
A B S T R A C T
Background: Although pharmacogenetics for major depressive disorder (MDD) is gaining momentum, the role of genetics in differences in response to antidepressant treatment is controversial, as they depend on multifactorial and polygenic phenotypes. Previous studies focused on the genes of the serotonergic system, leaving apart other pathological factors such as the inflammatory pathway. The main objective of the study was to assess whether treatment response might be associated with specific inflammationrelated genetic variants or their methylation status.

Methods: 41 SNPs in 8 inflammatory genes: interleukin (IL) 1- $\beta$, IL2, IL6, IL6R, IL10, IL18, tumor necrosis factor (TNF)- $\alpha$ and interferon (IFN)- $\gamma$ were genotyped in 153 patients with MDD, who were evaluated with the Mausdley Staging Method to determine treatment response profiles. Pyrosequencing reactions and methylation quantification were performed in a PyroMark Q24 in 5 selected CpG islands of IL1- $\beta$, IL6 and IL6R. Linear and logistic regression analyses were conducted, including age and gender as covariates using PLINK 1.07.

Results: Allelic distribution of IL1- $\beta$ rs 1143643 was significantly associated with MSM scores (FDR corrected $\mathrm{p}=0.04$ ). Allelic distribution of IL6R rs57569414 showed a trend towards significance with MSM scores $(\mathrm{p}=0.002$; FDR corrected $\mathrm{p}=0.07)$. Haplotype analyses showed associations between allelic combinations of IL1- $\beta$ and IL10 with treatment response (FDR corrected $\mathrm{p}<0.01$ ). Methylation percentage of treatment responders was only higher in an IL6R CpG island $(\mathrm{p}<0.05)$.

Conclusions: These exploratory findings suggest that IL1- $\beta$ and, marginally, IL6R polymorphisms may affect treatment response in major depression. If confirmed, these results may account for the heterogeneous phenotypes of major depression that underlie differences in treatment response.
\end{abstract}

(c) 2019 Elsevier Masson SAS. All rights reserved.

\section{Introduction}

Major Depressive Disorder (MDD) is one of the leading causes of disability worldwide affecting over 300 million people. [1] Around $30 \%$ of patients suffering from MDD do not respond to antidepressant treatment and are diagnosed with treatment resistant depression (TRD) after 2 unsuccessful courses of

\footnotetext{
* Corresponding author at: Department of Psychiatry, Institut d'Investigació Biomèdica Sant Pau, Hospital de la Santa Creu i Sant Pau, Av. Sant Antoni M. Claret, 167, 08025, Barcelona, Catalonia, Spain.

** Corresponding author at: Research Laboratory, Fundació Docència i Investigació Mútua de Terrassa, Sant Antoni, 32, 08221, Terrassa, Barcelona, Catalonia, Spain.

E-mail addresses: mjarranz@mutuaterrassa.es (M.J. Arranz), mportella@santpau.cat (M.J. Portella).
}

treatment with the appropriate dosage [2,3]. Treatment failure has a negative effect on patients' quality of life, is associated with increased risk for suicidal behavior and has higher economic costs for society [4]. In order to improve the efficacy of antidepressants, efforts have been made to identify biomarkers to predict treatment response, particularly within drug targets, i.e. serotonin pathways $[5,6]$. However, the findings to date have not fulfilled expected impact possibly because an individual's response to pharmacotherapy is multifactorial and involves a complex interplay of both genetic and environmental factors. [7]

Staging TRD has several benefits such as better predicting chances of future remission and guiding clinical treatment selection, minimizing the time that non-responders are on ineffective treatment. Various staging models of treatment resistance have been proposed. The Maudsley Staging Method 
for treatment resistant depression (MSM) developed by Fekadu and colleagues [8] has several advantages compared to previous models such as incorporating additional clinical information of duration and severity of TRD, in addition to its straightforwardness and clarity of usage. Moreover, Ruhé et al. [9] investigated several staging models for TRD and concluded that MSM has the most accurate predictive utility. This approach points towards the necessity of detecting treatment response profiles as soon as possible.

Genetic factors contribute around 35\% to the disease risk in MDD, [10] and are presumed to also explain differences in treatment response [11]. Several genes have been associated with treatment response in MDD, including the serotonin transporter (SLC6A4), and BDNF genes (Val66Met) amongst others. [12,13] Additionally, previous studies have identified genetic variants in hepatic metabolic enzymes (CYPs) and in several polymorphisms within serotonin genes associated with response to treatment with antidepressant drugs [14,15]. However, compelling evidence has identified inflammation as a promising etiopathological pathway of depression in the last 20 years. Activated inflammatory response has been detected in MDD [16]. Cytokines have been consistently shown to be elevated in depressed patients [17] and clinical similarities have been described between inflammatory diseases and MDD such as anhedonia, cognitive impairment and sleep disturbances [18]. As pro-inflammatory cytokines can decrease the production of serotonin, it has been proposed that medications blocking or reducing pro-inflammatory cytokines or those increasing anti-inflammatory cytokines might have therapeutic effects in patients with MDD (see [18] for a comprehensive review on targeting cytokines to reduce depressive symptoms). The underlying mechanism is that inflammatory cytokines can increase the expression and activity of monoamine transporters, the main targets of SSRIs antidepressants, [19] and decrease the levels of tetrahydrobiopterin, a cofactor essential for the synthesis of serotonin [20], but few studies have examined inflammatory polymorphisms as predictors of treatment response. In this regard, interleukin (IL) $1-\beta$ and IL6 has shown the most promising results as linked to treatment response, with $r s 1143643$ and $r s 16944$ [21,22] as prime candidates of IL1- $\beta$, and rs1800795 for IL6. [23] Other inflammatory candidate genes such as IL2, IL10, IL18, tumor necrosis factor alpha (TNF- $\alpha)$ and interferon-gamma (IFN- $\gamma$ ) have received less attention, although having been previously related with major depressive disorder. [24,25] Taking into account that response to medication may be a polygenic and complex trait than previously hypothesized and distinct molecular mechanisms seem to modulate inflammatory pathways as stated above [26], studies should address the role of different cytokine genetic variants, as response to medication may be as complex as the pathology. Besides, recent studies have also shown that alterations in the methylation status that regulate gene expression of these polymorphisms may contribute to disease risk and prognosis
$[27,28]$, but few studies have examined whether inflammatory genes are differentially methylated in depression (Ryan's study in late life depression [29]), or the association of methylation status of such polymorphisms with treatment outcomes.

The main aim of this study was to investigate the contribution of genetic variants and methylation status in inflammatory genes to treatment response in MDD patients.

\section{Methods}

\subsection{Sample}

The study was approved by the Research Ethics Committee of Hospital Sant Pau in Barcelona and was carried out in accordance with the Declaration of Helsinki. A total of 153 psychiatric outpatients who met the Diagnostic and Statistical Manual of the American Psychiatric Association (DSM-IV-TR) criteria for MDD were recruited. Psychiatric comorbidities were also screened following DSM-IV-TR criteria. The diagnoses were based on clinical interviews by senior psychiatrists and double-checked through clinical reports. To be included in the study, participants had to be 18 years or older, right handed and native Catalan and/or Spanish speakers (please note that Catalan inhabitants are mostly bilingual for these two languages). The exclusion criteria were: clinically significant physical or neurological disease (brain trauma with loss of consciousness) and mental retardation (score $<70$ on the estimated IQ using the Vocabulary subtest of the Wechsler Adult Intelligence Scale-IV, Spanish validated version). Participants were of Caucasians of European descent and all gave informed consents after a full explanation of the study protocol and that they can withdraw at any moment. They received no financial compensation for study participation. A blood sample was obtained from each individual $(10 \mathrm{ml})$ after inclusion in the study. All patients were on standard antidepressant treatment at the time of blood sampling, following the clinical guidelines of the national health system. In summary, $34 \%$ of patients were on monotherapy with SSRIs, $49 \%$ of patients were on combined treatments including SSRI or TCA plus lithium or benzodiazepines, and $17 \%$ of patients were on polytherapy, which included antidepressants (SSRIs, TCA and/or MAOIs), mood stabilizers (other than lithium) and/or antipsychotics. All medication was prescribed at therapeutic doses and for a sufficient time before changing or combining treatment strategies.

Table 1 summarizes the demographics and clinical characteristics of the study participants. A total of 56 patients had Axis I comorbidities, following the criteria of the Diagnostic and Statistical Manual of Mental Disorders (DSM-IV), - affective disorder (55\%), anxiety disorder (23\%), psychotic disorder (11\%) and schizo-affective disorder (11\%). Data for smoking habits was available for 119 patients, with 22 smokers in the non-resistant group and 16 in the resistant. Detailed information of illness course

Table 1

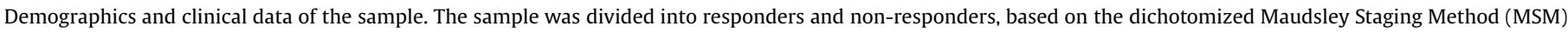
scores as follows: $M S M<=7$ were considered responders; MSM > 7, non-responders.

\begin{tabular}{|c|c|c|c|c|c|c|}
\hline & Total sample $(n=153)$ & Responders $(n=91)$ & Non-responders $(n=62)$ & Missing data & F or $\chi^{2}$ & p-value \\
\hline Gender (females) & $105(69 \%)$ & $58(64 \%)$ & $45(76 \%)$ & - & 2.495 & 0.080 \\
\hline Smokers & $38(25 \%)$ & $22(24 \%)$ & $16(26 \%)$ & $34(22 \%)$ & & \\
\hline Axis I \% & $56(37 \%)$ & $31(34 \%)$ & $25(40 \%)$ & - & & \\
\hline Age & $50.72(14.39)$ & $48.23(14.26)$ & $54.38(13.91)$ & - & 0.172 & 0.679 \\
\hline Age at onset & $38.14(12.38)$ & $38.46(11.71)$ & $37.68(13.39)$ & - & 2.974 & 0.087 \\
\hline HDRS-17 & $15.29(8.09)$ & $11.98(7.92)$ & $20.11(5.51)$ & $18(12 \%)$ & 10.24 & 0.002 \\
\hline MSM & $6.14(3.21)$ & $3.96(1.58)$ & $9.34(2.12)$ & - & 12.47 & 0.001 \\
\hline N. of depressive episodes & $2.47(1.87)$ & $1.85(0.92)$ & $3.37(2.45)$ & - & 17.86 & 0.001 \\
\hline
\end{tabular}

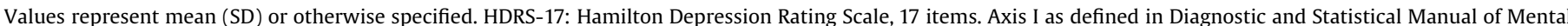
Disorders (DSM-IV). P-values considered significant at $<0.05$. 
and treatment response was also available. Severity of depression was assessed by the Hamilton Depression Rating Scale, 17 items (HDRS-17). [30] Illness stage was evaluated with the Maudsley Staging Method (MSM) [31], which included duration of index episode, symptom severity and treatment failures. This scale delivers a total score ranging from 3 to 15. For the purpose of the present study, and to better interpret the associations and to obtain odds ratios, MSM scores were also dichotomized between treatment non-response (MSM $>7 ; \mathrm{n}=62$ ) and response $(\mathrm{MSM}<=$ 7; $n=91$ ). After dividing the participants into responder and nonresponder groups, the first consisted of 33 males (mean age: 44.03) and 58 females (mean age: 51.04 ), while in the second there were 15 males (mean age: 51.71) and 47 females (mean age: 54.73) respectively. Differences between the two groups were tested with Chi-square test for gender; and with independent t-tests for age, age at onset, number of depressive episodes, HDRS-17 and MSM scores. Expectedly, there were significant differences only in number of episodes, HDRS-17 and MSM scores $(\mathrm{p}<0.05)$.

\subsection{DNA isolation}

Blood samples were systematically collected from the subjects upon admittance to the study. Genomic DNA was automatically extracted from peripheral whole-blood samples (Autopure, Qiagen, Hilden, Germany).

\subsection{Genetic studies}

A total of 41 Tag single nucleotide polymorphisms (SNPs) in 8 inflammatory genes (IL1- $\beta$, IL2, IL6, IL6R, IL10, IL18, TNF- $\alpha$ and IFN$\gamma$ ) were selected for genotyping using the HapMap programme (www.hapmap.org). The SNP selection criteria were: minor allele frequency (MAF) over 0.05 and $\mathrm{r} 2$ threshold of 0.8 in Caucasians. The SNPs were analyzed by real-time PCR using OpenArray $\mathbb{R}$ technology on the QuantStudio ${ }^{\mathrm{TM}} 12 \mathrm{~K}$ Flex Real-Time (Applied Biosystems, Foster City, CA, USA). Standard quality controls (>95\% genotyping success per individual and SNPs, Hardy-Weinberg equilibrium) were applied to the genotyping results.

\subsection{Methylation analyses}

Specific CpG sites in the $5^{\prime}$ regulatory region of selected genes (see Table 2 for details) were assessed by bisulfite-pyrosequencing. PyroMark Assay Design Software v.2.0 (QIAGEN, Hilden, Germany) was used to design the set of primers for PCR amplification and sequencing. Information on $\mathrm{CpG}$ sites locations and primers used is shown in Table 2. DNA bisulfite treatment and PCR amplification were performed by means of EpiTech Bisulfite kit and the PyroMark PCR kit (QIAGEN, Hilden, Germany) respectively, following the manufacturer recommendations. Pyrosequencing reactions and methylation quantification were performed in a PyroMark Q24 (QIAGEN, Hilden, Germany).

\subsection{Statistical analyses}

Linear and logistic regression analyses for genotype results were conducted considering MSM as the dependent variable (as continuous and binary factors respectively). A logistic regression analysis was performed for methylation results, using binary MSM distribution. Age and gender of patients were included as covariates in all analyses. Statistical analyses were performed using PLINK (version 1.07, Purcel et al., 2007). Significance was set at $\mathrm{p}<0.05$. Correction for multiple comparisons in allelic and genotype associations was carried out with false discovery rate (FDR).

\section{Results}

\subsection{Genetic results}

All investigated polymorphisms were in the Hardy-Weinberg equilibrium and showed a genotyping success $>95 \%$. Single marker analysis revealed uncorrected significant associations with the total MSM scores. The allelic distribution of an IL6R rs57569414 polymorphism was associated with MSM total scores (OR=-1.62; $\mathrm{p}=0.002$ ). Uncorrected significant associations were also found between MSM total scores and IL18 rs543810, IL1- $\beta$ rs1143643, IL6 rs2069824 and IFN- $\gamma$ rs2069718. Results from the allele association analysis are presented in Table 3. Analyses of genotype frequencies revealed nominal associations between the IL6 rs2069824, IL6R rs4075015, IL2 rs1479923 and IL10 rs3021094 polymorphisms and MSM scores ( $\mathrm{p}$ values $<0.05$ in all comparisons). Results from the genotype analysis are displayed in Table 4. Finally, uncorrected significant associations between haplotype combinations of the IL1- $\beta$, IL6 and IL10 polymorphisms investigated were also observed ( $\mathrm{p}$ values $=0.02,0.03$ and 0.03 , respectively).

Logistic regression models (i.e., responders vs non-responders) revealed uncorrected associations between the allelic and genotype distribution of the IL1- $\beta$ rs 1143643 polymorphisms and response to treatment $(\mathrm{OR}=2.49, \mathrm{p}=0.0009$; and $\mathrm{OR}=2.79$, $\mathrm{p}=0.002$, respectively). The allele and genotype frequency distribution of the IL6R rs4075015 polymorphism were also associated with MSM as a binary variable $(\mathrm{p}=0.04$ and $\mathrm{p}=0.03$, respectively). The allele distribution of IL6R $r s 57569414(\mathrm{p}=0.009)$, IL18 rs543810 ( $\mathrm{p}=0.03)$ and IFN- $\gamma$ rs1861493 $(\mathrm{p}=0.046)$ were also significantly associated with treatment resistance. Additionally, the genotype distribution of IL2 rs10027390 was nominally associated with treatment response $(p=0.05)$. Results from the genotype analysis are displayed in Table 4. Haplotype analyses revealed associations between allelic combinations of the genes IL1- $\beta$, IL10, IFN- $\gamma$ and IL6R with treatment response (uncorrected $\mathrm{p}=0.0006, \mathrm{p}=0.003, \mathrm{p}=0.03$ and $\mathrm{p}=0.05$, respectively).

Most of the associations vanished after FDR correction. Only the allelic association of IL1- $\beta$ rs 1143643 with MSM scores remained significant and the genotype association of IL1- $\beta$ showed a trend

Table 2

PCR amplification and pyrosequencing's design.

\begin{tabular}{|c|c|c|c|c|}
\hline Gene & Number of $\mathrm{CpG}$ sites & Primer ID & Primer sequence ( $5^{\prime}$ to $3^{\prime}$ ) & Localization (start-end) \\
\hline \multirow[t]{3}{*}{ IL6 } & 5 & $\mathrm{~F}$ & AGTGTAGGAAATTTTTAGTTTTGGAATTGT & $5139-5258$ \\
\hline & & $\mathrm{R}$ & АСАСААСТАAАAАССТАССТСТАСТАСТАА & \\
\hline & & $\mathrm{S}$ & AATTTTTAGTTTTGGAATTGTT & \\
\hline \multirow[t]{3}{*}{ IL6R } & 6 & $\mathrm{~F}$ & GGAGGGTTGGGGTAGTTAG & $5544-5887$ \\
\hline & & $\mathrm{R}$ & AACAATCTCCССТTAAAATAACCT & \\
\hline & & $\mathrm{S}$ & ACAATCCTATACACAAACC & \\
\hline \multirow[t]{3}{*}{ IL1- $\beta$} & 2 & $\mathrm{~F}$ & ATGGAAGGGTAAGGAGTAGTAA & 9195-9366 \\
\hline & & $\mathrm{R}$ & ATATCTTCСАСТTТАТСССАСАТАТ & \\
\hline & & $\mathrm{S}$ & ATGTAAATATGTATTGTTTTTTTGA & \\
\hline
\end{tabular}

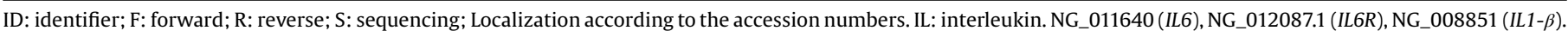


Table 3

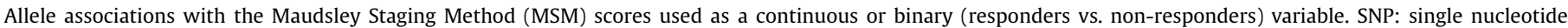
polymorphism. FDR: false discovery rate. Significant associations are presented in bold. IFN: interferon; IL: interleukin; TNF: tumor necrosis factor.

\begin{tabular}{|c|c|c|c|c|c|c|c|c|c|}
\hline \multirow[b]{2}{*}{ Gene } & \multirow[b]{2}{*}{ SNP } & \multicolumn{3}{|c|}{ MSM Continuous } & \multirow[b]{2}{*}{ (FDR) } & \multicolumn{3}{|c|}{ MSM Binary } & \multirow[b]{2}{*}{ (FDR) } \\
\hline & & Beta & $95 \% \mathrm{CI}$ & p-value & & OR & $95 \% \mathrm{CI}$ & p-value & \\
\hline IFN- $\gamma$ & rs1861493 & -0.68 & & 0.09 & & 0.56 & $0.32-0.99$ & 0.05 & $(0.37)$ \\
\hline IFN- $\gamma$ & rs2069718 & -0.69 & $-1.35--0.03$ & 0.04 & $(0.33)$ & 0.74 & & 0.20 & \\
\hline IFN- $\gamma$ & rs2069727 & 0.56 & & 0.11 & & 1.12 & & 0.63 & \\
\hline IL $1-\beta$ & rs1143634 & -0.16 & & 0.70 & & 0.70 & & 0.22 & \\
\hline IL $1-\beta$ & rs1143643 & 0.86 & $0.17-1.58$ & 0.02 & $(0.29)$ & 2.49 & $1.45-4.29$ & 0.0009 & $(0.04)$ \\
\hline IL1- $\beta$ & rs16944 & -0.34 & & 0.34 & & 0.69 & & 0.14 & \\
\hline IL2 & rs10027390 & -0.58 & & 0.11 & & 0.66 & & 0.11 & \\
\hline IL2 & rs1479923 & 0.70 & & 0.06 & & 1.39 & & 0.19 & \\
\hline IL2 & rs2069772 & -0.04 & & 0.93 & & 1.14 & & 0.65 & \\
\hline IL2 & rs2069778 & -0.16 & & 0.77 & & 0.84 & & 0.63 & \\
\hline IL2 & rs3136534 & -0.05 & & 0.89 & & 0.99 & & 0.98 & \\
\hline IL6 & rs10242595 & 0.57 & & 0.14 & & 1.21 & & 0.47 & \\
\hline IL6 & rs12700386 & -0.16 & & 0.74 & & 1.04 & & 0.91 & \\
\hline IL6 & rs1800795 & -0.15 & & 0.70 & & 1.05 & & 0.86 & \\
\hline IL6 & rs1800797 & -0.17 & & 0.66 & & 1.00 & & 1.00 & \\
\hline IL6 & rs2069824 & 1.04 & $0.04-2.04$ & 0.04 & $(0.33)$ & 1.55 & & 0.21 & \\
\hline IL6 & rs2069835 & 1.13 & & 0.07 & & 1.88 & & 0.13 & \\
\hline IL6 & rs2069837 & 0.52 & & 0.41 & & 1.05 & & 0.91 & \\
\hline IL6 & rs2069840 & -0.39 & & 0.32 & & 0.89 & & 0.66 & \\
\hline IL6R & rs12047973 & 0.30 & & 0.53 & & 0.99 & & 0.99 & \\
\hline IL6R & rs12083537 & -0.21 & & 0.66 & & 0.77 & & 0.43 & \\
\hline IL6R & rs3887104 & 0.44 & & 0.33 & & 1.23 & & 0.49 & \\
\hline IL6R & rs4075015 & 0.68 & & 0.05 & & 1.64 & $1.02-2.65$ & 0.04 & $(0.37)$ \\
\hline IL6R & rs4133213 & 0.06 & & 0.87 & & 0.93 & & 0.78 & \\
\hline IL6R & rs4556347 & -0.32 & & 0.42 & & 0.78 & & 0.35 & \\
\hline IL6R & rs57569414 & -1.62 & $-2.61--0.63$ & 0.002 & $(0.07)$ & 0.35 & $0.16-0.77$ & 0.009 & $(0.19)$ \\
\hline IL6R & rs6690230 & 0.10 & & 0.80 & & 1.20 & & 0.50 & \\
\hline IL10 & rs61815632 & 0.10 & & 0.83 & & 1.15 & & 0.67 & \\
\hline IL10 & rs1518110 & -0.52 & & 0.22 & & 1.01 & & 0.97 & \\
\hline IL10 & rs1554286 & -0.71 & & 0.12 & & 0.83 & & 0.55 & \\
\hline IL10 & rs1800890 & 0.32 & & 0.40 & & 1.59 & & 0.07 & \\
\hline IL10 & rs3021094 & -0.14 & & 0.79 & & 1.01 & & 0.98 & \\
\hline IL10 & rs3024505 & 0.13 & & 0.81 & & 1.46 & & 0.27 & \\
\hline IL18 & rs543810 & 1.32 & $0.25-2.04$ & 0.02 & $(0.29)$ & 2.28 & $1.08-4.79$ & 0.03 & $(0.37)$ \\
\hline IL18 & rs1946518 & 0.56 & & 0.12 & & 1.28 & & 0.30 & \\
\hline IL18 & rs3882891 & 0.31 & & 0.36 & & 1.21 & & 0.41 & \\
\hline TNF- $\alpha$ & rs1799724 & -0.48 & & 0.43 & & 0.74 & & 0.48 & \\
\hline TNF- $\alpha$ & rs1799964 & 0.14 & & 0.72 & & 1.10 & & 0.72 & \\
\hline TNF- $\alpha$ & rs1800629 & 0.21 & & 0.71 & & 1.10 & & 0.81 & \\
\hline TNF- $\alpha$ & rs3093664 & 0.08 & & 0.91 & & 1.13 & & 0.79 & \\
\hline TNF- $\alpha$ & rs361525 & -0.01 & & 0.99 & & 1.12 & & 0.81 & \\
\hline
\end{tabular}

towards significance (see Tables 3 and 4, respectively). Haplotype analyses for allelic combinations of IL1- $\beta$ and IL10 with binary MSM scores survived FDR correction ( $\mathrm{p}=0.004$ and $\mathrm{p}=0.01$, respectively).

\subsection{Epigenetic results}

None of the thirteen analyzed CpG sites in the IL6, IL6R and IL1- $\beta$ genes showed statistically significant differences in the methylation status when MSM score was used ( $\mathrm{p}>0.05$ in all comparisons). However, when comparing the methylation percentage of treatment responders and non-responders, one of the $\mathrm{CpG}$ sites in the IL6R gene showed a trend towards significance (1.7 vs. 1.5, respectively; $\mathrm{p}=0.05$, uncorrected for multiple testing). Results are summarized in Table 5.

\section{Discussion}

The current study investigated the influence of genetic alterations in inflammatory genes in relationship with treatment response in MDD. Inflammation is thought to play a role in the pathogenesis of depression, but the effect of inflammatory pathways on treatment response is still unclear. Our findings suggest that other factors beyond the serotonergic system may be involved in treatment response in major depression. In our study,
IL1- $\beta$ polymorphism showed consistent association with treatment response as measured with the MSM. IL6 and IL6R polymorphisms were marginally associated, although significance did not survive correction for multiple comparisons.

The association of IL1- $\beta$ rs 1143643 is in agreement with a previous study by Baune and colleagues [21] who reported two IL1$\beta$ polymorphisms ( $r s 1143643$ and $r s 16944$ ) to be related to SSRI treatment resistance. Another study by Yu and co-workers [22] also reported that the IL-1 $\beta$ rs16944 polymorphism was associated with poorer outcome after 4 weeks of treatment with fluoxetine. However, we were not able to replicate this finding in our study. This might be due to the fact that in this study patients were on a strict 4-week treatment with fluoxetine, while our patients were on a variety of antidepressants and were assessed after a long period of treatment. In addition, the different ethnicity of the participants may also account for the discrepancy. Nevertheless, we observed a significant association between IL1- $\beta$ haplotype combinations and treatment response.

With regards to IL6, the current results suggest that the rs2069824 polymorphism might be associated with treatment response, although these findings should be taken cautiously as were uncorrected for multiple comparisons. In any case, the rs2069824 allelic and genotype association with MSM suggest that this variant might be a prime candidate for future investigation. 
Table 4

Genotype associations with the Maudsley Staging Method (MSM) scores used as a continuous or binary (responders vs. non-responders) variable. SNP: single nucleotide polymorphism. Significant associations are presented in bold. IFN: interferon; IL: interleukin; TNF: tumor necrosis factor.

\begin{tabular}{|c|c|c|c|c|c|c|c|}
\hline \multirow[b]{2}{*}{ Gene } & \multirow[b]{2}{*}{ SNP } & \multicolumn{3}{|c|}{ MSM Continuous } & \multicolumn{3}{|c|}{ MSM Binary } \\
\hline & & Stat & $P$ value & (FDR) & Stat & $P$ value & (FDR) \\
\hline IFN- $\gamma$ & rs1861493 & 3.51 & 0.17 & & 4.36 & 0.11 & \\
\hline IFN- $\gamma$ & rs2069718 & 4.77 & 0.09 & & 4.37 & 0.11 & \\
\hline IFN- $\gamma$ & rs2069727 & 2.92 & 0.23 & & 0.23 & 0.89 & \\
\hline IL1- $\beta$ & rs1143634 & 0.21 & 0.89 & & 1.64 & 0.44 & \\
\hline IL1- $\beta$ & rs1143643 & 10.58 & 0.005 & $(0.1)$ & 12.79 & 0.002 & $(0.08)$ \\
\hline IL1- $\beta$ & rs16944 & 0.96 & 0.62 & & 2.36 & 0.31 & \\
\hline IL2 & rs10027390 & 3.96 & 0.14 & & 4.20 & 0.12 & \\
\hline IL2 & rs1479923 & 4.23 & 0.12 & & 4.84 & 0.09 & \\
\hline IL2 & rs2069772 & 0.04 & 0.98 & & 0.37 & 0.83 & \\
\hline IL2 & rs2069778 & 0.89 & 0.64 & & 0.27 & 0.87 & \\
\hline IL2 & rs3136534 & 0.02 & 0.99 & & 0.29 & 0.86 & \\
\hline IL6 & rs10242595 & 3.10 & 0.21 & & 0.75 & 0.69 & \\
\hline IL6 & rs12700386 & 0.13 & 0.94 & & 0.41 & 0.81 & \\
\hline IL6 & rs1800795 & 0.26 & 0.88 & & 0.03 & 0.98 & \\
\hline IL6 & rs1800797 & 0.82 & 0.66 & & 0.13 & 0.94 & \\
\hline IL6 & rs2069824 & 6.93 & 0.03 & $(0.41)$ & 0.14 & 0.93 & \\
\hline IL6 & rs2069835 & 3.69 & 0.16 & & 1.64 & 0.44 & \\
\hline IL6 & rs2069837 & 0.41 & 0.52 & & 0.001 & 0.98 & \\
\hline IL6 & rs2069840 & 1.04 & 0.59 & & 0.51 & 0.77 & \\
\hline IL6R & rs12047973 & 3.92 & 0.14 & & 0.18 & 0.55 & \\
\hline IL6R & rs12083537 & 0.77 & 0.68 & & 0.02 & 0.99 & \\
\hline IL6R & rs3887104 & 0.97 & 0.62 & & 0.48 & 0.79 & \\
\hline IL6R & rs 4075015 & 4.65 & 0.09 & & 4.71 & 0.09 & \\
\hline IL6R & rs4133213 & 0.44 & 0.80 & & 0.12 & 0.94 & \\
\hline IL6R & rs4556347 & 2.15 & 0.34 & & 1.11 & 0.57 & \\
\hline IL6R & rs57569414 & 10.61 & 0.005 & $(0.1)$ & 5.59 & 0.06 & \\
\hline IL6R & rs6690230 & 3.26 & 0.19 & & 2.15 & 0.34 & \\
\hline IL10 & rs61815632 & 0.61 & 0.74 & & 1.07 & 0.59 & \\
\hline IL10 & rs1518110 & 2.11 & 0.35 & & 0.84 & 0.66 & \\
\hline IL10 & rs1554286 & 3.86 & 0.15 & & 1.25 & 0.54 & \\
\hline IL10 & rs1800890 & 0.77 & 0.68 & & 3.21 & 0.20 & \\
\hline IL10 & rs3021094 & 5.13 & 0.08 & & 0.11 & 0.95 & \\
\hline IL10 & rs3024505 & 0.37 & 0.83 & & 1.82 & 0.40 & \\
\hline IL18 & rs543810 & 6.43 & 0.04 & $(0.41)$ & 5.92 & 0.05 & $(0.61)$ \\
\hline IL18 & rs1946518 & 3.68 & 0.16 & & 1.44 & 0.49 & \\
\hline IL18 & rs3882891 & 2.75 & 0.25 & & 1.22 & 0.54 & \\
\hline TNF- $\alpha$ & rs1799724 & 1.07 & 0.59 & & 0.15 & 0.93 & \\
\hline TNF- $\alpha$ & rs1799964 & 0.42 & 0.81 & & 0.52 & 0.77 & \\
\hline TNF- $\alpha$ & rs1800629 & 0.17 & 0.92 & & 0.001 & 0.99 & \\
\hline TNF- $\alpha$ & rs3093664 & 2.97 & 0.23 & & 0.09 & 0.96 & \\
\hline TNF- $\alpha$ & rs361525 & 0.003 & 0.99 & & 0.02 & 0.88 & \\
\hline
\end{tabular}

We did not find association with any of the other IL6 polymorphisms investigated. Carvalho and colleagues [23] had previously reported increased risk for resistance for patients having the rs1800795-“G/G" genotype. But, methodological differences such as study design and patients' selection could be underneath this discrepancy. In any case, both studies point towards the fact that genetic variations within cytokine genes, such as IL6 may be involved in antidepressant treatment outcomes.

Interestingly, we also found IL6R gene to be related to treatment response -rs4075015 and rs57569414 emerged as possible predictors in both the allele and genotype analyses, together with a marginal haplotype combination-. As the pro-inflammatory signaling of IL6 depends on the soluble IL6R [32], it can be suggested that these genetic variants might play a role in this process. Our exploratory epigenetic study revealed marginal difference in IL6R methylation, with responders showing a higher methylation than non-responders, suggesting that an alteration in expression of the receptor gene may be associated with fluctuations in IL6 peripheral levels (see for example [33] reporting repeatedly increase plasma levels of IL6 in patients with worse treatment prognosis). Previous studies suggested that methylation changes might occur in MDD patients. An Epigenome wide study (EWAS) conducted in post-mortem frontal cortex from MDD patients revealed alterations in the methylation status of 224 regions, with differences $>10 \%$. [34]. Recently, several studies have suggested that the methylation status of genes such as SLC6A4, NR3C1, BDNF and IL6 may constitute peripheral biomarkers for MDD. [35-37,29], Uddin and co-workers [38] reported an inverse correlation between the level of methylation of IL6 CpGs and circulating IL6 and levels in patients with lifetime depression. We observed additional associations between pro-inflammatory and anti-inflammatory variants and treatment response. Previous research has suggested that pro-inflammatory IL18 variants might play a role in MDD. Haastrup and colleagues [39] reported association between the IL18 rs1946518 variant and increased risk of depression in patients with past stressful life events. However, in our study we did not find any significant association with this polymorphism. Nevertheless, we observed the IL18 rs543810 allelic distribution associated with MSM when treated as total score and as binary variable, with $\mathrm{OR}>2$, although such associations did not survive FDR correction. To date, no other study has linked this polymorphism with treatment response, thus the relevance of this finding may also be spurious.

Despite the increasing interest in the inflammation theory of MDD, previous genetic research on cytokines has focused mainly on IL1- $\beta$, IL6 and IL18 variants. In light of our findings, the rest of studied cytokines, i.e., IL2, IFN- $\gamma$ and IL10 were not clearly associated with treatment response in our sample of MDD, but larger studies should explore the involvement of these variants in treatment outcomes. IL10 haplotype combinations were associated with response in our study. A study by Song and colleagues [40] suggested decreased levels of IL10 in depressed patients after 6 weeks of treatment and our results supports the notion that IL10 might be associated with worse treatment prognosis. With all the

Table 5

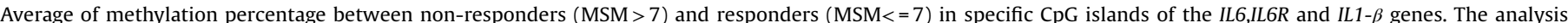

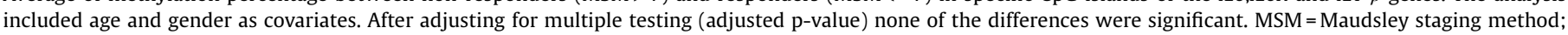
$\mathrm{FC}=$ fold change; IL: interleukin.

\begin{tabular}{|c|c|c|c|c|c|}
\hline $\mathrm{CpG}$ & Non- Responders (MSM > 7) & Responders $(\mathrm{MSM}<=7$ ) & LogFC & $\mathrm{t}$ & p-value \\
\hline IL6_1 & 3.516833 & 3.411333 & 0.04 & 0.15 & 0.88 \\
\hline IL6_2 & 4.726102 & 4.622889 & 0.13 & 0.21 & 0.83 \\
\hline IL6_3 & 2.874068 & 2.765 & 0.02 & 0.09 & 0.92 \\
\hline IL6_4 & 7.173158 & 6.930449 & -0.08 & -0.19 & 0.85 \\
\hline IL6_5 & 6.008772 & 5.750341 & -0.11 & -0.29 & 0.82 \\
\hline IL6R_1 & 1.423559 & 1.47686 & 0.08 & 0.72 & 0.47 \\
\hline IL6R_2 & 1.647627 & 1.757647 & 0.13 & 1.34 & 0.18 \\
\hline IL6R_3 & 1.890169 & 2.015 & 0.12 & 1.40 & 0.16 \\
\hline IL6R_4 & 1.5152 & 1.693714 & 0.20 & 1.94 & 0.05 \\
\hline IL6R_5 & 1.292881 & 1.214217 & -0.03 & -0.22 & 0.82 \\
\hline IL6R_6 & 1.812182 & 1.980125 & 0.18 & 1.49 & 0.14 \\
\hline IL $1-\beta \_1$ & 7.120333 & 7.583933 & 0.14 & 0.38 & 0.70 \\
\hline IL $1-\beta \_2$ & 9.466207 & 9.94236 & 0.20 & 0.46 & 0.64 \\
\hline
\end{tabular}


reserves due to the exploratory nature of the present study, these findings suggest that not only pro, but also anti-inflammatory cytokines may influence treatment response. The contribution of a single polymorphism or a haplotype to the peripheral cytokine levels is not clear, although a previous study reported a correlation between a IL1- $\beta$ haplotype and a 2-3-fold increase in the secretion levels. [41] Our results might be in line with the previously described mechanism linking increased pro- and anti-inflammatory cytokine levels with worse tratment response. Nevertheless, we did not measure the peripheral serum levels of the investigated cytokines and therefore these conclusions remain speculative.

The present study has several limitations that need to be considered when interpreting the results. It possesses the classical limitations of a candidate genes study - the sample size is moderate and results should be confirmed in a larger independent study, as previous research exploring an already published statistically significant finding for a SNP has often failed to reproduce those findings, implying a large number of false-positive reports. Another pitfall is that it is unclear whether the SNPs have functionally significant effects on the gene or they are simply useful markers. Nonetheless, together with genome wide association studies, candidate gene studies still provide important information about disease mechanisms and the ability to predict individuals who are at risk. A second limitation is that we did not control for differences in antidepressants' type and dose taken by participants. In any case, the naturalistic nature of the present study still provides realistic information about patients that become treatment resistant. A third possible limitation is that information about experienced childhood stressful events was not collected -a factor previously linked to aberrant methylatione. [42] Finally, plasma levels of specific cytokines would have added useful information to check the hypothesis of the study. In any case, a replication of these findings is warranted in future studies.

To conclude, the current findings support that treatment response might be associated with specific genetic variants, and partly by the methylation status, of the inflammation-related gene IL1- $\beta$ and, to a lesser extent, IL6 and IL6R. If confirmed, these results can provide information on additional genetic markers of response and constitute putative new targets for future novel therapies.

\section{Conflict of interests}

J.D.A received consultancy and/or lecture honoraria from Lundbeck, Pfizer, Neuraxpharm and Janssen in the last year, none of them with direct relation with this work. Thre rest of authors declare no conflict of interests related with this work.

\section{Funding}

This study was supported by an Intramural (IIBSP-2015-83), and partly funded by the European Regional Development Fund (ERDF) and by CERCA Programme (Generalitat de Catalunya). M.J.P. is funded by the Ministerio de Ciencia e Innovación of the Spanish Governement and by the Instituto de Salud Carlos III through a "Miguel Servet II" research contract (CP16/00020; PI16/00302), from the National Research Plan de I+D+I 2016-2019, co-financed by the ERDF.

\section{Acknowledgements}

We would like to thank all the patients for their selfless participation in this study.

\section{References}

[1] World Health Organization. Depression and other common mental disorders. Who; 2017. p. 24.
[2] Rush AJ, Trivedi MH, Wisniewski SR, et al. Acute and longer-term outcomes in depressed outpatients requiring one or seve. Am J Psychiatry 2006;163:1905-17.

[3] Conway CR, George MS, Sackeim HA. Toward an evidence-based, operational definition of treatment-resistant depression: when enough is enough. JAMA Psychiatry 2017;74:9-10.

[4] Mrazek DA, Hornberger JC, Altar CA, Degtiar I. A review of the clinical, economic, and societal burden of treatment-resistant depression: 1996-2013. Psychiatr Serv 2014;65:977-87.

[5] Miller JM, Hesselgrave N, Ogden RT, et al. Brain serotonin 1A receptor binding as a predictor of treatment outcome in major depressive disorder. Biol Psychiatry 2013;74:760-7.

[6] Helton SG, Lohoff FW. Serotonin pathway polymorphisms and the treatment of major depressive disorder and anxiety disorders. Pharmacogenomics 2015; $16: 541-53$.

[7] Amare AT, Schubert KO, Klingler-Hoffmann M, Cohen-Woods S, Baune BT. The genetic overlap between mood disorders and cardiometabolic diseases: a systematic review of genome wide and candidate gene studies. Transl Psychiatry 2017;7:e1007-12.

[8] Fekadu A, Wooderson SC, Markopoulou K, Cleare AJ. The maudsley staging method for treatment-resistant depression: prediction of longer-term outcome and persistence of symptoms. J Clin Psychiatry 2009;70:952-7.

[9] Ruhé HG, van Rooijen G, Spijker J, FPML Peeters, Schene AH. Staging methods for treatment resistant depression. A systematic review. J Affect Disord 2012;137:35-45.

[10] Geschwind DH, Flint J. Genetics and genomics of psychiatric disease HHS public access. Science (80-) 2015;349:1489-94.

[11] Uher R, Tansey KE, Henigsberg N, et al. Common genetic variation and antidepressant efficacy in major depressive disorder: a meta-analysis of three genome-wide pharmacogenetic studies. Am J Psychiatry 2013;170:207-17.

[12] Keers R, Uher R, Huezo-Diaz P, et al. Interaction between serotonin transporter gene variants and life events predicts response to antidepressants in the GENDEP project. Pharmacogenomics J 2011;11:138-45.

[13] Niitsu T, Fabbri C, Bentini F, Serretti A. Pharmacogenetics in major depression: a comprehensive meta-analysis. Prog Neuro-Psychopharmacol Biol Psychiatry 2013;45:183-94.

[14] Fabbri C, Serretti A. Pharmacogenetics of the efficacy and side-effects of antidepressant drugs. Genetic influences on response to drug treatment for major psychiatric disorders. . p. 39-55.

[15] Fabbri C, Crisafulli C, Calabrò M, Spina E, Serretti A. Progress and prospects in pharmacogenetics of antidepressant drugs. Expert Opin Drug Metab Toxicol 2016;12:1157-68.

[16] Dantzer R, O'Connor JC, Freund GG, Johnson RW, Kelley KW. From inflammation to sickness and depression: when the immune system subjugates the brain. Nat Rev Neurosci 2008;9:46.

[17] Rosenblat JD, Cha DS, Mansur RB, McIntyre RS. Inflamed moods: a review of the interactions between inflammation and mood disorders. Prog NeuroPsychopharmacology Biol Psychiatry 2014;53:23-34.

[18] Shariq AS, Brietzke E, Rosenblat JD, Barendra V, Pan Z, McIntyre RS. Targeting cytokines in reduction of depressive symptoms: a comprehensive review. Prog Neuro-Psychopharmacol Biol Psychiatry 2018;83:86-91.

[19] Miller AH, Maletic V, Raison CL. Inflammation and its discontents: the role of cytokines in the pathophysiology of major depression. Biol Psychiatry 2009;65:732-41.

[20] Haroon E, Raison CL, Miller AH. Psychoneuroimmunology meets neuropsychopharmacology: translational implications of the impact of inflammation on behavior. Neuropsychopharmacology 2012;37:137-62.

[21] Baune BT, Dannlowski U, Domschke K, et al. The interleukin 1 Beta (IL1B) Gene Is associated with failure to achieve remission and impaired emotion processing in Major depression. Biol Psychiatry 2010;67:543-9.

[22] Yu YWY Chen TJ, Hong CJ, Chen HM, Tsai SJ. Association study of the interieukin-Ibeta (C-5I IT) genetic polymorphism with major depressive disorder, associated symptomatology, and antidepressant response. Neuropsychopharmacology 2003;28:1182-5.

[23] Carvalho S, Santos M, Lima L, et al. IL6-174G \& C genetic polymorphism influences antidepressant treatment outcome. Nord J Psychiatry 2017;71:15862.

[24] Mihailova S, Ivanova-Genova E, Lukanov T, Stoyanova V, Milanova V, et al. A study of TNF- $\alpha$, TGF- $\beta$, IL-10, IL- 6 , and IFN- $\gamma$ gene polymorphisms in patients with depression. J Neuroimmunol 2016, doi:http://dx.doi.org/10.1016/j. jneuroim.2016.03.005.

[25] Shelton RC, Claiborne J, Sidoryk-Wegrzynowicz M, et al. Altered expression of genes involved in inflammation and apoptosis in frontal cortex in major depression. Mol Psychiatry 2011, doi:http://dx.doi.org/10.1038/mp.2010.52.

[26] Malki K, Tosto MG, Mouriño-Talín H, et al. Highly polygenic architecture of antidepressant treatment response: comparative analysis of SSRI and NRI treatment in an animal model of depression. Am J Med Genet Part B Neuropsychiatr Genet 2017, doi:http://dx.doi.org/10.1002/ajmg.b.32494.

[27] Lisoway AJ, Zai CC, Tiwari AK, Kennedy JL. DNA methylation and clinical response to antidepressant medication in major depressive disorder: a review and recommendations. Neurosci Lett 2018;669:14-23.

[28] Bufalino C, Hepgul N, Aguglia E, Pariante CM. The role of immune genes in the association between depression and inflammation: a review of recent clinical studies. Brain Behav Immun 2013;31:31-47.

[29] Ryan J, Pilkington L, Neuhaus K, Ritchie K, Ancelin ML, Saffery R. Investigating the epigenetic profile of the inflammatory gene IL-6 in late-life depression. BMC Psychiatry 2017; 17:354. 
[30] Hamilton M. A rating scale for depression. J Neurol Neurosurg Psychiatry 1960;23:56

[31] Fekadu A, Wooderson SC, Markopoulou K, Cleare AJ. The Maudsley Staging Method for treatment-resistant depression: prediction of longerterm outcome and persistence of symptoms. J Clin Psychiatry 2009;70:9527.

[32] Hodes GE, Ménard C, Russo SJ. Integrating Interleukin-6 into depression diagnosis and treatment. Neurobiol Stress 2016;4:15-22.

[33] Kiraly DD, Horn SR, Van Dam NT, et al. Altered peripheral immune profiles in treatment-resistant depression: response to ketamine and prediction of treatment outcome. Transl Psychiatry 2017;7:e1065.

[34] Sabunciyan S, Aryee MJ, Irizarry RA, et al. Genome-wide DNA methylation scan in major depressive disorder. PLoS One 2012;7:e34451.

[35] Januar V, Ancelin ML, Ritchie K, Saffery R, Ryan J. BDNF promoter methylation and genetic variation in late-life depression. Transl Psychiatry 2015;5:e619-7.

[36] Won E, Choi S, Kang J, et al. Association between reduced white matter integrity in the corpus callosum and serotonin transporter gene DNA methylation in medication-naive patients with major depressive disorder. Transl Psychiatry 2016;6:e866-9.
[37] Roy B, Shelton RC, Dwivedi Y. DNA methylation and expression of stress related genes in PBMC of MDD patients with and without serious suicidal ideation. J Psychiatr Res 2017;89:115-24.

[38] Uddin M, Koenen KC, Aiello AE, Wildman DE, De Los Santos R, Galea S. Epigenetic and inflammatory marker profiles associated with depression in a community-based epidemiologic sample. Psychol Med 2011;41:997-1007.

[39] Haastrup E, Drachmann J, Bock C, et al. Promoter variants in IL18 are associated with onset of depression in patients previously exposed to stressful-life events. J Affect Disord 2012;136:134-8.

[40] Song C, Halbreich U, Han C, Leonard BE, Luo H. Imbalance between pro-and anti-inflammatory cytokines, and between Th1 and Th2 cytokines in depressed patients: the effect of electroacupuncture or fluoxetine treatment. Pharmacopsychiatry 2009;42:182-8.

[41] Hall SK, Perregaux DG, Gabel CA, et al. Correlation of polymorphic variation in the promoter region of the interleukin- $1 \beta$ gene with secretion of interleukin$1 \beta$ protein. Arthritis Rheum 2004;50:1976-83.

[42] Essex MJ, Boyce WT, Hertzman C, Lam LL, Armstrong JM. Epigenetic vestiges of early developmental adversity: childhood stress exposure and DNA methylation in adolescence marilyn. Child Dev 2014;84:58-75. 